\title{
Patología maligna tiroidea. Hospital Sabogal, Callao
}

\author{
Juan Oré ${ }^{1}$, Marco Otárola ${ }^{2}$ \\ ${ }^{\text {I} P o l i c l i ́ n i c o ~ N a v a l, ~ M a r i n a ~ d e ~ G u e r r a ~ d e l ~ P e r u ́ . ~}{ }^{2}$ Servicio de Cirugía de Cabeza y Cuello, \\ Hospital Alberto Sabogal Sologuren, Callao, Perú.
}

\begin{abstract}
Resumen
OBJETIVOS: Determinar las características clínicas del cáncer de tiroides encontrados en la población del Callao. DISEÑO: Estudio descriptivo retrospectivo. MATERIAL Y MÉTODOS: Se revisó las historias clínicas de las intervenciones quirúrgicas de la glándula tiroides en el Hospital Nacional Alberto Sabogal Sologuren (HNASS), en el periodo enero de 2000 a diciembre de 2002, cuyo resultado por anatomía patológica fue compatible con neoplasia maligna de dicha glándula. RESULTADOS: Las 45 neoplasias malignas primarias de tiroides encontradas representaron $34,2 \%$ de la patología tiroidea operada en el periodo en estudio. El carcinoma papilar se presentó en $100 \%$ de los pacientes de sexo masculino y en $82 \%$ del sexo femenino; en la mujer, los carcinomas folicular, medular y linfoma representaron $10,3 \%, 5,1 \%$ y $2,6 \%$, respectivamente. El grupo etáreo con mayor incidencia fue entre 50 y 69 años, con una edad promedio de 53 años. Dentro de los diferentes tipos de neoplasias malignas, el más frecuente fue el papilar con 38 casos $(84,4 \%)$, seguido de los carcinomas folicular y medular con $8,9 \%$ y 4,4\%, respectivamente. El síntoma más frecuente fue el aumento de volumen (61,5\%), seguido de dolor $(15,4 \%)$, disfagia $(10,8 \%)$ y disfonía $(9,2 \%) ; 29,6 \%$ se encontraba en estadio I y $59,1 \%$ en estadio II; sólo 11,4\% estaba en estadio III. CONCLUSIONES: El predominio de casos en mujeres del carcinoma papilar y la clínica asociada, fueron acordes con lo descrito en la literatura.
\end{abstract}

Palabras clave: Neoplasmas de la tiroides; carcinoma papilar; carcinoma medular.

\section{Malignant thyroid pathology. Callao, Sabogal Hospital Summary}

OBJECTIVES: To determine the clinical characteristics of thyroid cancer at Callao's population. DESIGN: Descriptive and retrospective study. MATERIAL AND METHODS: Clinical histories and surgical reports that involved the thyroid gland were reviewed. The pathology result had to be compatible with primary thyroid malignant neoplasm. The study was performed at the Alberto Sabogal Sologuren National Hospital (HNASS) during the period January 2000 through December 2002. RESULTS: Forty-five thyroid malignant neoplasms were identified representing $34,2 \%$ of all thyroid neoplasms operated during the period studied. In males papillary carcinoma was present in $100 \%$ of cases and in females in $82,1 \%$, followed by both follicular and medullar carcinoma and lymphoma, in 10,2\%, 5,1\% and 2,6\%, respectively. Major incidence was in the 50 to 69 years group, average 53 years. The most frequent malignant neoplasms were papillary carcinoma with 38 cases $(84,4 \%)$, follicular and medullary carcinoma with $8,9 \%$ and $4,4 \%$, respectively. Most frequent symptoms were growing tumor $(61,5 \%)$, local pain $(15,4 \%)$, dysphagia $(10,8 \%)$ and dysphonia (9,2\%); $29,6 \%$ of cases were on stage I, $59,1 \%$ on stage II, and only $11,4 \%$ on stage III. CONCLUSIONS: Papillary carcinoma occurred mainly in women and clinical features were as described in the literature.

Key words: Thyroid neoplasms; carcinoma, papillary; carcinoma, medullary.

\section{INTRODUCCIÓN}

La patología maligna tiroidea representa menos de $1 \%$ de los cánceres en general, pero el $90 \%$ de las patologías malignas endocrinas; ocasiona aproximadamente $0,4 \%$ de la mortalidad relacionada al cáncer

\footnotetext{
Correspondencia:

Dr. Juan Francisco Oré Acevedo

Av. Paseo la Castellana 620 Dpto. 102

Lima 33, Perú.

E-mail:juanfcoore@yahoo.com
} 
$\left({ }^{1-3}\right)$.

Cerca de 20 a $30 \%$ de los nódulos tiroideos y $5 \%$ de los bocios multinodulares resultan ser malignos. Es más frecuente en mujeres que en varones, relación aproximada de 3 a $1\left(^{1,2}\right)$.

En los años 2000 y 2001, en el Servicio de Cabeza y Cuello del Instituto Nacional de Enfermedades Neoplásicas la patología maligna en cabeza y cuello comprendió $9,3 \%$ y $8,1 \%$ del total de los pacientes en dichos años, situándose las neoplasias malignas de la tiroides en quinto lugar en el orden de frecuencia (entre 1995 y 1999), con $6,2 \%$ a $7,2 \%$ de los procesos malignos en dicha especialidad. Ocuparon el tercer lugar en mujeres entre los 15 a 39 años $(13,1 \%)$ y el sexto lugar, entre los 45 y 84 años $(4,4 \%)\left({ }^{4}\right)$.

La clínica más frecuente es una tumoración palpable, indurada e indolora, de crecimiento rápido. También se pueden presentar disfonía, fijación dérmica del tumor, disnea y disfagia, anorexia, disminución de peso; la función hormonal es variable. Las metástasis son raras e involucran ganglios regionales y luego en pulmones $(1,2,5,6)$

Podemos clasificar los procesos malignos de la glándula en primarios y secundarios, siendo los primeros los más frecuentes y de mayor importancia. Las neoplasias primarias se dividen según tengan células parenquimales (epiteliales) o del estroma glandular (fibrosarcomas, linfomas y hemangioendoteliomas malignos).

Entre los tumores epiteliales, tenemos las formas diferenciadas (carcinoma papilar y carcinoma folicular), formas intermedias (carcinoma medular y de células escamosas) y la indiferenciada (carcinoma anaplásico) $\left(^{1,7}\right)$.

Los carcinomas diferenciados corresponden a cerca de 90 a $95 \%$ de los carcinomas primarios $\left({ }^{4}\right)$.

El carcinoma papilar es el más frecuente y de menor agresividad de todos los carcinomas tiroideos, representando 76 a $80 \%$ de ellos. En cifras generales, sólo hacen $1,5 \%$ de todos los cánceres en adultos y $3 \%$ de todos los cánceres en niños $(2,5,8,9)$. La edad promedio es de 30 a 40 años, aunque $10 \%$ se desarrollan en menores de
20 años. Se presenta una relación de 3 a 1 en mujeres frente a varones $\left({ }^{1,5,6,8}\right)$.

Usualmente es un nódulo solitario indoloro de crecimiento lento, pero puede presentarse también como bocio multinodular; involucra ambos lóbulos tiroideos en un $30 \%\left({ }^{9,10,11}\right)$.

$\mathrm{Al}$ diagnóstico, 10 a $15 \%$ de los pacientes tiene metástasis a los huesos y los pulmones. $\left({ }^{1,2,6,8}\right)$.

Los valores de recurrencia están entre 15 y $25 \%$ de los pacientes; sin embargo, la sobrevida es alentadora, $90 \%$ a 20 años $(1,12,13)$.

El carcinoma folicular corresponde a $10 \%$ de las neoplasias malignas tiroideas, siendo más agresivo que el carcinoma papilar $(2,5,8,9)$.

La presentación es entre 40 y 50 años y más en mujeres, con una relación de 3 a 1 . Se presentan como nódulos solitarios de crecimiento variable, casi siempre indoloros. Raramente producen tirotoxicosis $(<1 \%)$, debido a que se presentan con mayor frecuencia en lugares con deficiencia de yodo en la dieta $(2,6,8)$.

Una variante aquí es el carcinoma de células Hürtle, el cual produce tiroglobulina. Son más multifocales o bilaterales y rara vez captan yodo y $25 \%$ ya tienen metástasis al diagnóstico $\left({ }^{1,2,9}\right)$.

El carcinoma medular representa entre 4 y $5 \%$ de los carcinomas de tiroides. Producen calcitonina con localización en la parte media a superior de los lóbulos tiroideos $\left.{ }^{(2,5,6,8}\right)$

No hay diferencia significativa según el sexo, con una relación mujeres-varones de 1,5-1 $\left({ }^{1,2,14}\right)$.

Cerca de $33 \%$ de ellos son familiares, siendo el restante esporádico; en ambas situaciones, se pueden encontrar asociados a la neoplasia endocrina múltiple tipo 2 (NEM-2) $\left(^{1,2,6,9}\right)$.

La edad típica de presentación en la forma esporádica es entre 50 y 60 años y en la forma asociada a la NEM-2 entre 20 y 30 años $(2,10)$.

Son unilaterales en $75 \%$ y la mayoría dolorosas, con disfonía, disfagia o disnea. Se asocian también 
disminución de peso, letargia y dolores óseos, diarrea o síndrome carcinoide $\left.{ }^{2,6}\right)$.

Sólo $1 \%$ de los carcinomas tiroideos corresponde al carcinoma anaplásico, siendo la mayoría de ellos originado de un tumor diferenciado $\left({ }^{2,5,9}\right)$

Tiene igual distribución según el sexo que el carcinoma medular, con mayor incidencia entre 60 y 80 años. Es un tumor de crecimiento indurado y rápido, haciéndolo el más agresivo de todos los carcinomas. Es frecuente el compromiso local, con disfonía, disfagia o disnea; además, presenta metástasis cercanas y a distancia $\left({ }^{2,6,9}\right)$.

Al diagnóstico, 50 a $69 \%$ de los pacientes tiene ya adenopatías evidentes $(7,9,14,15)$.

Dentro de los linfomas, predomina el no Hogdkin de células B, con una presentación cercana al $1 \%$ de las neoplasias malignas tiroideas. Confundible con tiroiditis, ya que se presentan luego o durante la tiroiditis de Hashimoto, se muestran como bocio multinodular o difuso, de crecimiento rápido, doloroso ${ }^{(2,5,8)}$.

Es más frecuente en mujeres que en varones, principalmente entre los 55 y 75 años $\left(^{6,16}\right)$.

Los otros tumores malignos son extraordinariamente infrecuentes. Las metástasis tiroideas son infrecuentes y son manifestación tardía de un cáncer no primario de la glándula. $\left(^{(2,5)}\right.$.

En cuanto al tratamiento, la tiroidectomía es el componente inicial y más importante en el manejo del carcinoma tiroideo, salvo el anaplásico. La tiroidectomía subtotal, con o sin istmectomía según el caso, es adecuada para pacientes con carcinomas no indiferenciados o microcarcinomas; mientras que la tiroidectomía total está indicada para el resto de carcinomas y recidivas locales $(2,5,7,17)$.

Se debe considerar la terapia posterior con $\mathrm{I}^{131}$, según el grado histológico del tumor y para metástasis sensibles o tejido tiroideo residual, seguida de terapia hormonal. De poseer metástasis ganglionares, se añade la disección radical modificada de cuello $\left({ }^{1,2,5,9,18}\right)$.

\section{MATERIAL Y MÉTODOS}

Se realizó un estudio descriptivo retrospectivo en el que se revisó todas las historias clínicas y los reportes operatorios de los pacientes sometidos a cirugía electiva de la glándula tiroides. La población se obtuvo del registro de altas del Servicio de Cirugía de Cabeza y Cuello del Hospital Nacional Alberto Sabogal Sologuren (HNASS) en el Callao, Lima, Perú, en el periodo comprendido entre enero 2000 y diciembre 2002.

Se incluyó todos los pacientes intervenidos, con resultados anatomopatológicos compatibles con neoplasia maligna tiroidea, en los cuales la pieza quirúrgica fue dicha glándula en su totalidad o en forma parcial.

Los criterios de exclusión incluyó aquellos pacientes sometidos a cirugía tiroidea con patología diferente de la catalogada como neoplasia maligna o al encontrar historias clínicas con datos incompletos, perdidos o inconsistentes; no hubo casos excluidos.

Se consideró las siguientes variables: edad, sexo, síntomas de ingreso, signos clínicos durante la exploración física, diagnóstico preoperatorio, operación realizada, complicaciones y resultado del informe anatomopatológico.

Se identificó las historias clínicas a revisar y se procedió a confeccionar un documento base para revisar las historias clínicas en el servicio de admisión y archivo del HNASS, siendo el levantamiento de datos llenado mediante el cuestionario en forma manual. Se elaboró una hoja de recolección de datos para los resultados obtenidos, siendo procesados con el programa estadístico de Microsoft Excel 2002.

\section{RESULTADOS}

De 190 cirugías de la glándula tiroides realizadas entre enero de 2000 y diciembre de 
2002, 45 tuvieron como resultado neoplasia maligna primaria de tiroides, correspondiendo a $34,2 \%$ del total de operaciones. La distribución según el sexo mostró un predominio para el sexo femenino, con $86,7 \%$ de la patología frente a $13,3 \%$ para el sexo masculino (Figura 1).

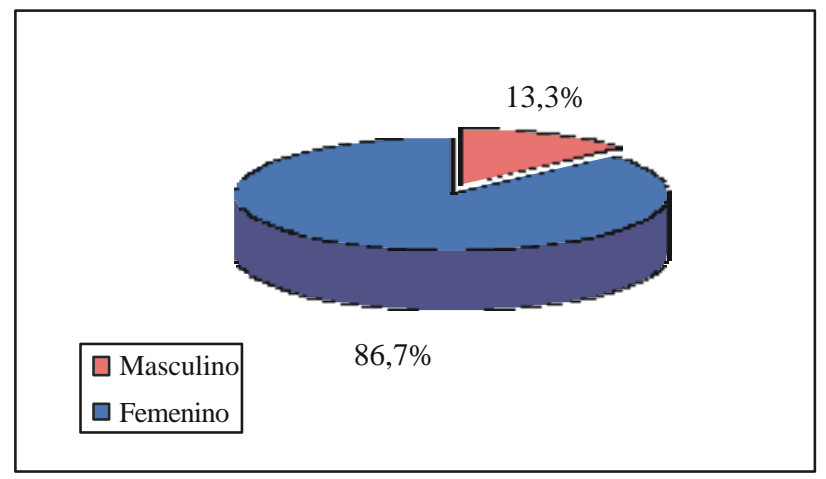

Figura 1.- Distribución de casos según sexo.

Dentro de los diferentes tipos de neoplasias malignas, el más frecuente fue el papilar, con 38 casos $(84,4 \%) ; 21 \%$ de ellos ya tenía metástasis. Le sigue el carcinoma folicular y medular, con $8,9 \%$ y $4,4 \%$, respectivamente, y el linfoma de células medianas a grandes 2,2\%. En ambos sexos, el carcinoma papilar fue el predominante, no habiendo otro carcinoma en el sexo masculino. Mientras que en el sexo femenino, $82,1 \%$ correspondió a carcinoma papilar; siguieron en orden de frecuencia el carcinoma folicular, medular y linfoma, respectivamente (Tabla 1).

La edad promedio fue 53 años. El grupo etáreo con mayor incidencia fue entre 50 y 69 años, con $40 \%$ de los casos. Hubo sólo un caso en menores de 20 años y otro en el grupo de 80 a 89 años, ambos de carcinoma papilar de tiroides. El grupo etáreo con mayor presentación de carcinoma papilar fue 50 a 59 años, aunque se evidenció que no es predominante de estas edades, ya que, solamente entre los 30 y 60 años encontramos $52,3 \%$ de esta patología.

Para el carcinoma folicular, entre 50 y 69 años se encuentra el grupo con mayor cantidad de casos (75\%); el carcinoma medular se presentó entre los 60 y 79 años. El único caso de linfoma se presentó
Tabla 1.- Distribución de patologías según sexo.

\begin{tabular}{|c|c|c|c|c|c|c|}
\hline \multirow[b]{2}{*}{ AP } & \multicolumn{2}{|c|}{ Masculino } & \multicolumn{2}{|c|}{ Femenino } & \multicolumn{2}{|c|}{ Total } \\
\hline & $\mathrm{n}$ & $\%$ & $\mathrm{n}$ & $\%$ & $\mathrm{n}$ & $\%$ \\
\hline Papilar & 6 & 100,0 & 32 & 82,1 & 38 & 84,4 \\
\hline Folicular & 0 & 0,0 & 4 & 10,3 & 4 & 8,9 \\
\hline Medular & 0 & 0,0 & 2 & 5,1 & 2 & 4,4 \\
\hline Linfoma & 0 & 0,0 & 1 & 2,6 & 1 & 2,2 \\
\hline Total & 6 & 100,0 & 39 & 100,0 & 45 & 100,0 \\
\hline
\end{tabular}

a la edad de 65 años (Figura 2).

En el cuadro clínico de los pacientes en estudio, el aumento del volumen glandular es el signo y síntoma predominante $(61,5 \%)$, seguido del dolor $(15,4 \%)$. El resto de pacientes reportó dolor, disfagia y disfonía en 10, 7 y 6 casos, respectivamente. Sólo un paciente presentó clínica de hipotiroidismo $(1,5 \%)$ y otro palpitaciones $(1,5 \%)$, ambos en carcinomas papilares. En cuanto a las otras neoplasias, dos casos presentaron disfagia, uno por carcinoma folicular y otro por carcinoma medular; el linfoma se asoció a dolor y disfonía. El resto de la clínica manifestada se encontró en el carcinoma papilar (Tabla 2).

De los carcinomas intervenidos, más de $50 \%$ de ellos estaba en el estadio II, encontrándose a carcinoma folicular y medular sólo en este estadio. En el carcinoma papilar, sólo $16,2 \%$ de los casos

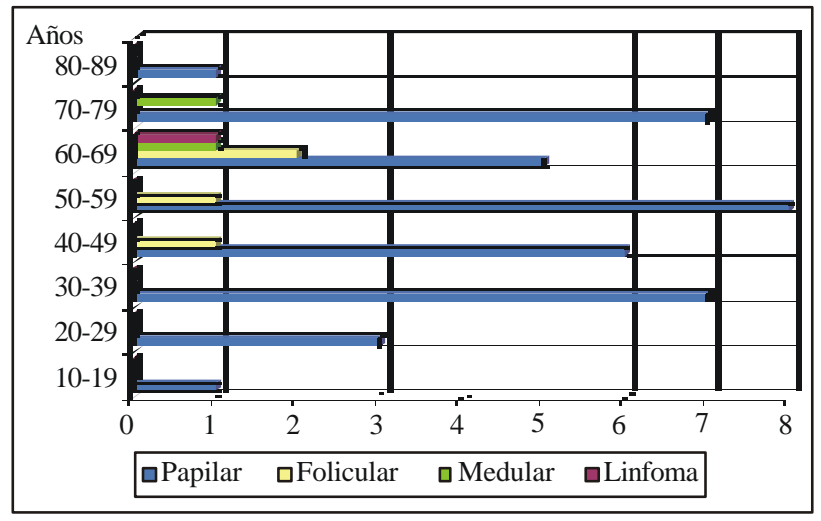

Figura 2.- Distribución de patologías según grupos etáreos. 
Tabla 2.- Distribución de la clínica asociada.

\begin{tabular}{lrr}
\hline \multirow{2}{*}{ Clínica } & \multicolumn{2}{c}{ Total } \\
\cline { 2 - 3 } & $\mathrm{n}$ & \multicolumn{1}{c}{$\%$} \\
\hline Tumor & 40 & 61,5 \\
Dolor & 10 & 15,4 \\
Disfagia & 7 & 10,8 \\
Disfonía & 6 & 9,2 \\
Palpitaciones & 1 & 1,5 \\
Hipotiroidismo & 1 & 1,5 \\
Total & 65 & 100,0 \\
\hline
\end{tabular}

estaba en estadio III (Tabla 3).

\section{DISCUSIÓN}

El sexo femenino es el predominante para todos los carcinomas y linfomas, con relación de 5,3/1 sobre los varones en el carcinoma papilar de tiroides y de 6,5/1 para los procesos malignos en general, entre mujeres y varones. El promedio de edad fue 53 años.

En la Tabla 1 observamos que $100 \%$ de los casos malignos para varones fue el carcinoma papilar; mientras que, para las mujeres el carcinoma predominante fue también el papilar con $82,1 \%$, y menos del $19 \%$ restante se repartió entre el carcinoma folicular, medular y el linfoma primario de tiroides.

El carcinoma papilar se mantiene sobre el resto de procesos malignos y con predominio entre los 30 y 60 años. La incidencia del carcinoma folicular es menor de $10 \%$ del total y en pacientes de 40 a
70 años; mientras que, la frecuencia del carcinoma medular y linfoma entra en los rangos de frecuencia esperados $(8,9 \%$ y $4,5 \%$, respectivamente) y en la presentación de pacientes mayores de 60 años.

Respecto de la clínica asociada, el signo más relevante fue la tumoración de la glándula tiroides $(61,5 \%)$ y de ellas, $15,4 \%$ se asoció a dolor. Si bien se presentó disfagia y disfonía, no fueron en tal cantidad como se esperaría encontrar en los procesos malignos de la tiroides, al igual que otros síntomas. Así, en 44,4\% (20/45) de pacientes se presentó una tumoración indolora que finalmente fue catalogada como neoplasia maligna de la tiroides.

La intervención quirúrgica que más se realizó fue la tiroidectomía total en $40 \%$ de los pacientes, siguiéndole en frecuencia, la tiroidectomía subtotal y la tiroidectomía total más disección radical modificada bilateral de cuello, en $20 \%$ para cada una de ellas (Tabla 4). Hubo 8 casos de carcinoma papilar en los cuales, por metástasis ganglionar, se realizó adicionalmente una disección de cuello.

Tres pacientes fueron intervenidos por segunda vez por patologías no malignas de tiroides.

Del estadiaje de los pacientes intervenidos, la mayoría de los casos se encontró en estadio I y II, con $29,6 \%$ y $59,1 \%$, respectivamente, todos ellos sin metástasis a distancia; sólo $11,4 \%$ se encontraba en estadio III; no hubo casos en estadio IV.

\section{AGRADECIMIENTOS}

A la gerencia del Hospital Nacional Alberto Sabogal

Tabla 3.- Estadiaje según el sistema TNM: tumor, ganglios y metástasis.

\begin{tabular}{|c|c|c|c|c|c|c|c|}
\hline \multirow[t]{2}{*}{ Estadio } & \multicolumn{2}{|c|}{ I } & \multicolumn{2}{|c|}{ II } & \multicolumn{2}{|c|}{ III } & \multirow[t]{2}{*}{ Total } \\
\hline & $\mathrm{n}$ & $\%$ & $\mathrm{n}$ & $\%$ & $\mathrm{n}$ & $\%$ & \\
\hline Cáncer papilar & 13 & 34,2 & 20 & 52,6 & 5 & 16,2 & 38 \\
\hline Cáncer folicular & 0 & 0,0 & 4 & 100,0 & 0 & 0,0 & 4 \\
\hline Cáncer medular & 0 & 0,0 & 2 & 100,0 & 0 & 0,0 & 2 \\
\hline Total & 13 & 29,6 & 26 & 59,1 & 5 & 11,4 & 44 \\
\hline
\end{tabular}


Tabla 4.- Cirugías realizadas según patología.

\begin{tabular}{|c|c|c|c|c|c|c|c|}
\hline \multirow[t]{2}{*}{ Cirugía } & \multirow[t]{2}{*}{ Papilar } & \multirow[t]{2}{*}{ Folicular } & \multirow[t]{2}{*}{ Medular } & \multirow[t]{2}{*}{ Linfoma } & \multirow{2}{*}{$\begin{array}{c}\text { Papilar } \\
+ \text { metástasis }\end{array}$} & \multicolumn{2}{|c|}{ Total } \\
\hline & & & & & & $\mathrm{n}$ & $\%$ \\
\hline Tiroidectomía subtotal & 7 & 2 & 0 & 0 & 0 & 9 & 20,0 \\
\hline Tiroidectomía complementaria & 2 & 0 & 0 & 0 & 0 & 2 & 4,4 \\
\hline Tiroidectomía total (TT) & 12 & 2 & 2 & 0 & 1 & 17 & 37,8 \\
\hline $\mathrm{TT}+$ disección recurrencial & 1 & 0 & 0 & 0 & 0 & 1 & 2,2 \\
\hline TT + DRM unilateral cuello & 4 & 0 & 0 & 0 & 2 & 6 & 13,3 \\
\hline TT + DRM bilateral cuello & 3 & 0 & 0 & 1 & 5 & 9 & 20,0 \\
\hline $\mathrm{TT}+$ disección radical & 1 & 0 & 0 & 0 & 0 & 1 & 2,2 \\
\hline Total & 30 & 4 & 2 & 1 & 8 & 45 & 100,0 \\
\hline
\end{tabular}

DRM: Disección radical modificada.

Sologuren por haber permitido la realización del presente estudio; al servicio de Cirugía de Cabeza y Cuello del hospital en mención, por otorgar el apoyo necesario durante la investigación y para el Departamento de Admisión y de Patología del HNASS, sin los cuales no se hubiera podido llevar a cabo este estudio.

\section{BIBLIOGRAFÍA}

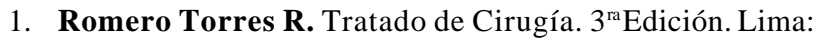
Medicina Moderna; 2000.

2. Swartz S. Principios de Cirugía. $7^{\text {ma }}$ Edición. México: McGraw-Hill; 2000.

3. Ariyan S. Cancer Of The Head And Neck. $1^{\text {st }}$ Edition. St. Louis: Mosby; 1987.

4. Instituto Nacional de Enfermedades Neoplásicas. Reporte de Neoplasias Malignas 1995 a 1999 e Indicadores Anuales de Gestión 2000 y 2001. Disponible en: http:// www.inen.sld.pe

5. Greenfield LJ, Mulholland MW, Oldhan KT. Head and neck. En: Surgery: Scientific Principles and Practice. $3^{\text {rd }}$ Edition. EUA: Editorial Lippincott Williams \& Wilkins; 2001

6. Fauci A, Martín J, Braunwald E, Kasper D. Harrison Principios de Medicina Interna. $14^{\text {ta }}$ Edición. Madrid: McGraw-Hill; 1998.

7. Niederhuber JE. Fundamentals of Surgery. $1^{\text {st }}$ Edition. EU de A: Lange; 1998

8. Cotran R, Kumar V, Robbins SL. Patología estructural y funcional. $5^{\text {ta }}$ Edición. Madrid: McGraw-Hill. 1990.

9. Way LW, Doherty GM. Current Surgical Diagnosis \& Treatment. $11^{\text {th }}$ Edition. EUA: Lange; 2003.

10. Myers EN, Suen JY. Cancer of the Head and Neck. $2^{\text {nd }}$ Edition. USA: Churchill Livingstone Inc; 1989.

11. Novelli JL, Piazza MV, Sánchez A.Patología Quirúrgica

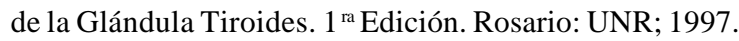

12. Dworkin HJ, Meier DA, Kaplan M. Advances in the management of patients with thyroid disease. Semin Nucl Med 1995; 25(3): 205-20.

13. Feld S, Garcia M. AACE Clinical Practice Guidelines For The Diagnosis And Management Of Thyroid Nodules; 1996.

14. Harvey HK.Diagnosis and management of the thyroid nodule. An overview. Otolaryngol Clin North Am 1990; 23(2): 30337.

15. Campbell JP, Pillsbury HC3 ${ }^{\text {rd }}$. Management of the thyroid nodule. Head Neck 1989; 11(5): 414-25.

16. Ashcraft MW, Van Herle AJ. Management of thyroid nodules. I: History and physical examination, blood tests, X-ray tests, and ultrasonography. Head Neck Surg 1981; 3(3): 216-30.

17. Correa P, Chen VW. Endocrine gland cancer. Cancer 1995; 75(1 Suppl): 338-52.

18. Hay ID, Bergstralh EJ, Goellner JR. Predicting outcome in papillary thyroid carcinoma. Surgery $1993 ; 114: 1050-8$. 